\title{
Fuzzy in 3-D: Contrasting Complex Fuzzy Sets with Type-2 Fuzzy Sets
}

\author{
Sarah Greenfield \\ Centre for Computational Intelligence (CCI) \\ School of Computer Science \& Informatics \\ De Montfort University \\ Leicester LE1 9BH, UK. \\ Email: s.greenfield@dmu.ac.uk
}

\author{
Francisco Chiclana \\ CCI and DMU Interdisciplinary Group in \\ Intelligent Transport Systems (DIGITS) \\ School of Computer Science \& Informatics \\ De Montfort University \\ Leicester LE1 9BH, UK. \\ Email: chiclana@dmu.ac.uk
}

\begin{abstract}
Complex fuzzy sets come in two forms, the standard form, postulated in 2002 by Ramot et al., and the 2011 innovation of pure complex fuzzy sets, proposed by Tamir et al.. In this paper we compare and contrast both forms of complex fuzzy set with type-2 fuzzy sets, as regards their rationales, applications, definitions, and structures. In addition, pure complex fuzzy sets are compared with type-2 fuzzy sets in relation to their inferencing operations.

Complex fuzzy sets and type-2 fuzzy sets differ in their roles and applications. Their definitions differ also, though there is equivalence between those of a pure complex fuzzy set and a type-2 fuzzy set. Structural similarity is evident between these three-dimensional sets. Complex fuzzy sets are represented by a line, and type-2 fuzzy sets by a surface, but a surface is simply a generalisation of a line. This similarity is particularly evident between pure complex fuzzy sets and type-2 fuzzy sets, which are both mappings from the domain onto the unit square. Type2 fuzzy sets were found not to be isomorphic to pure complex fuzzy sets.
\end{abstract}

\section{INTRODUCTION}

Complex fuzzy sets are a relatively recent development in fuzzy set theory, proposed by Ramot et al. in 2002 [1]. A natural extension of real-valued (type-1) fuzzy sets, they differ from them in so far as their membership grades are complex numbers (of modulus $<1$ ) [2].

Pure complex fuzzy sets are a very recent variation on complex fuzzy sets proposed by Tamir et al. in 2011. Using Cartesian coordinates, both the real and imaginary components of the membership grade may take any value within the interval $[0,1]$ [3, page 293]. Pure complex fuzzy sets may also be represented using polar coordinates [3, page 294] in a formalisation that is superficially similar to that of complex fuzzy sets as defined by Ramot et al., but in which the phase and modulus terms are interpreted differently.

In this paper, in order to distinguish between pure complex fuzzy sets and the original complex fuzzy sets proposed by Ramot et al., we shall refer to complex fuzzy sets as first postulated as standard complex fuzzy sets. The phrase 'complex fuzzy set' will refer to either form.

Type-2 fuzzy sets are another extension of type-1 fuzzy sets in which the sets' membership grades are themselves type-1 fuzzy sets. The concept dates back to Zadeh's seminal paper of 1975 [4].
The purpose of this paper is to establish similarities and differences firstly between complex fuzzy sets and type- 2 fuzzy sets, and secondly between complex fuzzy inferencing systems and type-2 fuzzy inferencing systems. The report is structured as follows: After the introduction of this section, Section II sets out the definitions of the fuzzy sets. In Section III, the structures of the sets are discussed, and in Section IV, the inferencing operations are investigated. The final section, Section V, concludes the paper.

\section{A. Fuzzy Inferencing Systems}

It is via the Fuzzy Inferencing System (FIS) ${ }^{1}$ that fuzzy sets are put to use. An FIS is a decision making program which works by applying fuzzy logic operators to commonsense linguistic rules. In this paper we are concerned with the Mamdani FIS, in which a crisp numerical input passes through three stages: fuzzification, inferencing, and finally defuzzification. The output of inferencing is a fuzzy set known as the aggregated set. During the defuzzification stage the aggregated set is converted into a crisp number, which is the output of the FIS. Figure 1 provides a representation of a Mamdani-style type-2 FIS. A Mamdani-style complex FIS differs from the type- 2 version only in that defuzzification is a one stage procedure. Defuzzification is beyond the scope of this paper; the focus will be on the inferencing stages of the FIS.

\section{B. Complex Fuzzy Sets}

Ramot et al. [1, page 171] see standard complex fuzzy sets as an extension of type-1 fuzzy sets:

“... complex fuzzy set theory modifies the original concept of fuzzy membership by asserting that, at least in some instances, it is necessary to add a second dimensions to the expression of membership. ... The novelty of complex fuzzy sets is manifested in the additional dimension of membership: the phase of the grade of membership, $\omega_{S}(x)$."

And what is the purpose of this additional phase term? It permits complex fuzzy sets to intuitively represent fuzziness

\footnotetext{
${ }^{1}$ A fuzzy inferencing system is also known as a Fuzzy Logic System (FLS).
} 
Type-2 FLS

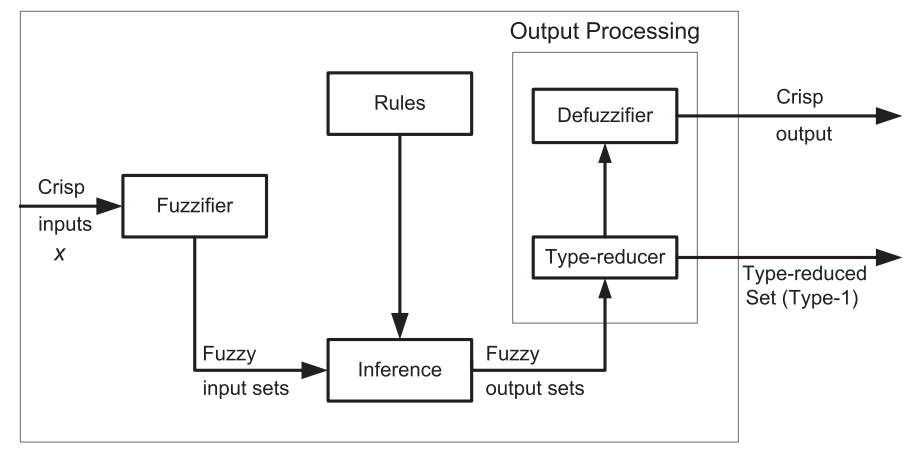

Fig. 1. Mamdani-style Type-2 FIS (adapted from Mendel [5]).

in time series applications. Dick gives this example [2, page 413]:

“... consider traffic congestion in a major city. The basic dynamics of traffic congestion are depressingly well-known: there is a morning "rush hour" when workers are trying to get to their jobs, causing high congestion in one direction on major roads; in the afternoon, there is a rush hour in the opposite direction, as everyone goes home. In between, traffic is lighter, and at night, the roads are nearly empty. This situation is approximately periodic, but never exactly repeats itself. Zadeh has recently [6] termed this phenomenon "regularity,"..."

Dick goes on to say that in his view,

“... the proper role for a complex fuzzy set is a remarkably efficient representation of approximately periodic phenomena, and as the underlying mathematical foundation of regularity."

Applications of complex fuzzy sets include analysing solar activity as measured by the recorded number of sunspots [1], Section III, signal processing [1], stock trading on the New York Stock Exchange [7], and prediction of voter turnout in elections [7]. The potential for further applications is immense.

\section{Type-2 Fuzzy Sets}

Type-1 membership functions are subject to uncertainty arising from various sources [8]. Their accuracy is therefore questionable; it seems counterintuitive to use real numbers, possibly expressed to several decimal places, to represent degrees of membership. Klir and Folger comment [9, page 12]:

“... it may seem problematical, if not paradoxical, that a representation of fuzziness is made using membership grades that are themselves precise real numbers. Although this does not pose a serious problem for many applications, it is nevertheless possible to extend the concept of the fuzzy set to allow the distinction between grades of membership to become blurred. Sets described in this way are known as type 2 fuzzy sets."
Here Klir and Folger describe blurring a type-1 fuzzy set to form an interval type-2 fuzzy set. Mendel and John take this idea a stage further [8, page 118], describing the transition from a type-1 fuzzy set to a generalised type-2 fuzzy set, again by blurring the type-1 membership function:

"Imagine blurring the type-1 membership function $[\ldots]$ by shifting the points $[\ldots]$ either to the left or the right, and not necessarily by the same amounts, [...]. Then, at a specific value of $x$, say $x^{\prime}$, there no longer is a single value for the membership function $\left(u^{\prime}\right)$; instead the membership function takes on values wherever the vertical line $\left[x=x^{\prime}\right]$ intersects the blur. These values need not all be weighted the same; hence, we can assign an amplitude distribution to all of these points. Doing this for all $x \in X$, we create a three-dimensional membership function a type- 2 membership function - that characterizes a type-2 fuzzy set."

Thus type-2 fuzzy sets take two forms, the interval, for which all secondary membership grades are 1 , and the generalised, where the secondary membership grade may take any value between 0 and 1 . The tendency has been for developers to opt $[10$, pages $7,8,16]$ for the computationally simpler interval type-2 FISs [8], [11] for which applications have been developed in areas such as control, simulation and optimisation [12], [13], [14], [15], [16], [17]. In contrast, there are relatively few, though varied, generalised type-2 fuzzy applications [18], [8], [19], [20]. Since strategies have been and continue to be developed that reduce the computational complexity of all stages of the generalised type-2 FIS [21], [22], [23], it is to be hoped that in the future there will be an increasing number of generalised type-2 FIS applications.

For a complex fuzzy set, the third dimension reflects additional information - that of phase. However, for a type-2 fuzzy set, the third dimension reflects the uncertainty arising out of a deficit in information.

\section{Definitions}

\section{A. Type-1 Fuzzy Sets}

Since complex fuzzy sets and type-2 fuzzy sets are both extensions of the basic type-1 fuzzy set, we begin by formally defining the type-1 fuzzy set.

Definition 1 (Type-1 Fuzzy Set). Let $X$ be a universe of discourse. A fuzzy set $A$ in $X$ is characterised by a membership function $\mu_{A}: X \rightarrow[0,1]$, and can be expressed as follows:

$$
A=\left\{\left(x, \mu_{A}(x)\right) ; \mu_{A}(x) \in[0,1] \forall x \in X\right\} .
$$

Note that the membership grades of $A$ are crisp, real numbers.

\section{B. Complex Fuzzy Sets}

Standard complex fuzzy sets are defined using polar coordinates.

Definition 2 (Standard Complex Fuzzy Set [1, page 172]). "A complex fuzzy set $S$, defined on a universe of discourse $U$, is 
characterized by a membership function $\mu_{S}(x)$ that assigns any element $x \in U$ a complex-valued grade of membership in $S$. By definition, the values $\mu_{S}(x)$ may receive all lie within the unit circle in the complex plane, and are thus of the form $r_{S}(x) \cdot e^{j \omega_{S}(x)}$, where $j=\sqrt{-1}, r_{S}(x)$ and $\omega_{S}(x)$ are both real-valued, and $r_{s}(x) \in[0,1]$.

The complex fuzzy set $S$ may be represented as the set of ordered pairs

$$
S=\left(x, \mu_{S}(x)\right) \mid x \in U^{\prime \prime}
$$

This definition employs polar coordinates. However conversion between the polar form and the Cartesian form is straightforward; in Figure 4(b) the data displayed is in Cartesian form.

Tamir et al. comment on this definition [3, page 286], "In the formalism of complex fuzzy sets proposed by Ramot et al., fuzzy membership is represented in polar coordinates and only the absolute value of the complex membership function conveys fuzzy information." In response, they define pure complex fuzzy sets which use "... a Cartesian complex fuzzy membership function where both the real part and the imaginary part can be fuzzy functions." [3, page 286]

Definition 3 (Pure Complex Fuzzy Set [3, pages 291-292]). Let $\mu(V, z)$ be the pure complex grade of membership. Then

$$
\mu(V, z)=\mu_{r}(V)+j \mu_{i}(z)
$$

where $\mu_{r}(V)$ and $\mu_{i}(z)$ are real value fuzzy grades of membership in the interval $[0,1]$.

\section{Type-2 Fuzzy Sets}

Let $X$ be a universe of discourse. Let $\tilde{P}(X)$ be the set of fuzzy sets in $X$. A type-2 fuzzy set $\tilde{A}$ in $X$ is a fuzzy set whose membership grades are themselves fuzzy. This implies that $\mu_{\tilde{A}}(x)$ is a fuzzy set in $[0,1]$ for all $x$, i.e.

$$
\tilde{A}=\left\{\left(x, \mu_{\tilde{A}}(x)\right) ; \mu_{\tilde{A}}(x) \in \tilde{P}([0,1]) \forall x \in X\right\} .
$$

It follows that $\forall x \in X \exists J_{x} \subseteq[0,1]$ such that $\mu_{\tilde{A}}(x): J_{x} \rightarrow$ $[0,1]$. Applying (1), we have:

$\mu_{A}(x)=\left\{\left(u, \mu_{\tilde{A}}(x)(u)\right) ; \mu_{\tilde{A}}(x)(u) \in[0,1] \forall u \in J_{x} \subseteq[0,1]\right\}$.

$J_{x}$ is called the primary membership of $x$ while $\mu_{\tilde{A}}(x)$ is called the secondary membership of $x$.

Putting (3) and (4) together we have

Definition 4 (Type-2 Fuzzy Set).

$$
\begin{aligned}
\tilde{A}=\left\{\left(x,\left(u, \mu_{\tilde{A}}(x)(u)\right)\right) \mid\right. & \mu_{\tilde{A}}(x)(u) \in[0,1], \\
& \left.\forall x \in X \wedge \forall u \in J_{x} \subseteq[0,1]\right\},
\end{aligned}
$$

where $X$ is a universe of discourse and $\tilde{A}$ is a type-2 fuzzy set in $X$.

Two concepts relating to type- 2 fuzzy sets are the footprint of uncertainty and the vertical slice.

Definition 5 (Footprint Of Uncertainty). The Footprint Of Uncertainty (FOU) is the projection of the type-2 fuzzy set onto the $x-u$ plane.
Definition 6 (Vertical Slice). A vertical slice is a plane which intersects the $x$-axis (primary domain) and is parallel to the $u$-axis (secondary domain).

The notion of a vertical slice may be extended to complex fuzzy sets in both the standard and pure forms.

\section{STRUCTURE}

Figure 2 shows a type-2 fuzzy set (from a Matlab ${ }^{T M}$ application), together with its FOU. Figure 3 shows the conventional 2-D representation of the time series consisting of sunspot numbers observed on a monthly basis [24]. Figure 4 shows the sunspot observations of Figure 3 [24] displayed as a complex fuzzy set.

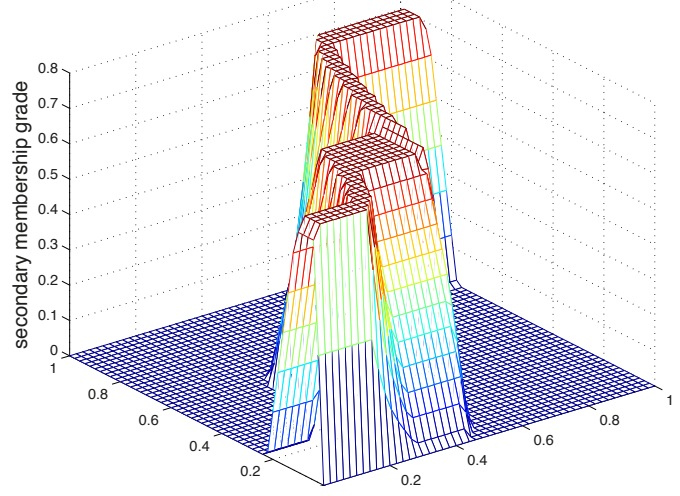

(a) 3-D representation

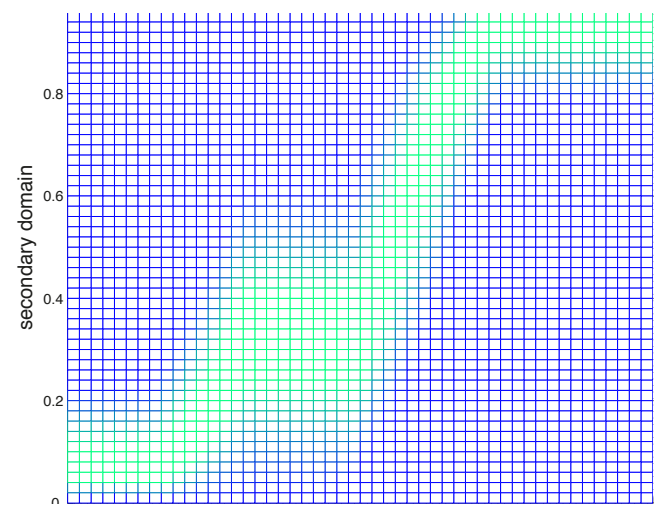

(b) FOU

Fig. 2. Aggregated type-2 fuzzy set created during the inference stage of a type-2 FIS.

A standard complex fuzzy set is represented mathematically by a mapping whose range is the unit disc, centre $(0,0)$. In contrast, the mapping representing a pure complex fuzzy set has the unit square, with vertices $(0,0),(1,0),(1,1),(0,1)$, as its range. Similarly, the range of the type-2 fuzzy set mapping is the unit square.

What is the difference in structure between a complex fuzzy set and a generalised type- 2 fuzzy set? Might not a complex fuzzy set be seen a special case of a generalised type- 2 fuzzy set? Graphically a type-2 fuzzy set is a surface in 3-D, whereas a complex fuzzy set is a line in 3-D. But a line is a specific sort of surface. So structurally a complex fuzzy set may be 


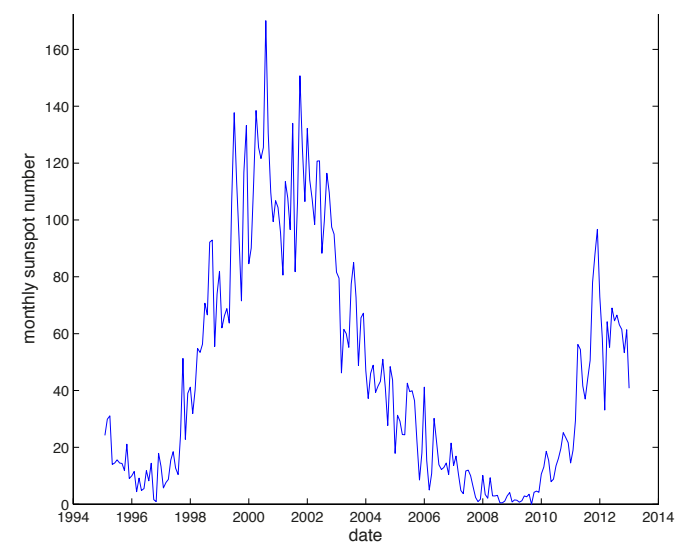

Fig. 3. Number of sunspots recorded on a monthly basis between 1994 and 2013.

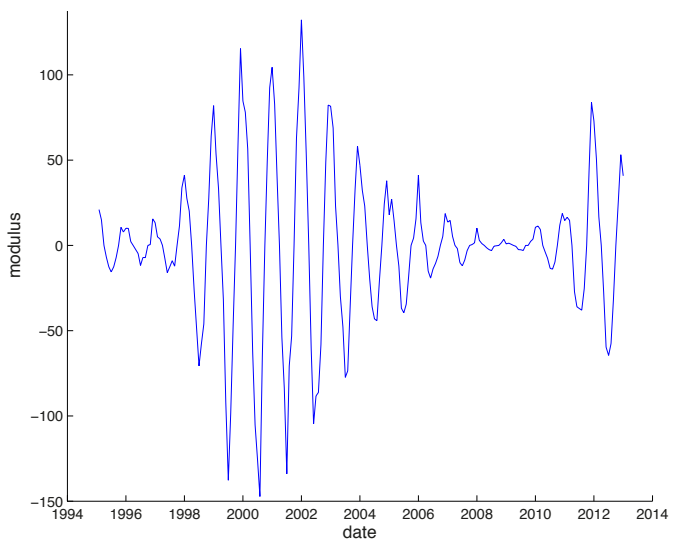

(a) 2-D view.

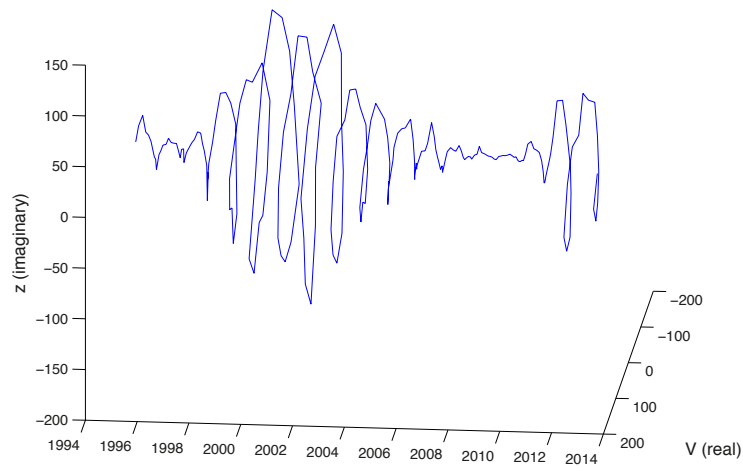

(b) 3-D view

Fig. 4. Sunspot data represented as a complex fuzzy set. The modulus is greater than 1 because the sunspot data has not been normalised. considered to be a special form of generalised type-2 fuzzy set (in the same way that an interval type- 2 fuzzy set is a specialisation of the generalised type-2 fuzzy set).

\section{OpERATIONS}

Within a type-2 FIS, join and meet operations are used extensively. Similarly, for a complex FIS, union and intersection operations are pervasive. For both type- 2 and complex FISs, all the computation occurring during the major stage of inferencing is founded upon these operations. In this section we will look at whether the type- 2 fuzzy join and meet operations are transferable to complex fuzzy sets.

To facilitate comparison between mappings, it is essential that they take the same range, otherwise a bijective transformation between the ranges would need to be exhibited. As this is not a trivial issue, we restrict our analysis to comparing type-2 fuzzy sets with pure complex fuzzy sets.

Minimum (' $\wedge$ ' or ' $\odot$ ') is the most frequently used t-norm; in the analysis which follows, this t-norm is employed, as is the maximum t-conorm ( $~ V$ ' or ' $\oplus$ ').

\section{A. Operations on Pure Complex Fuzzy Sets}

1) Equation for Union: According to Tamir et al. [3, pages 299-300] there are three ways to construct the union of two pure complex fuzzy classes ${ }^{2}$. They describe this (their third) construction as "... sound, intuitive, and practical."

Definition 7 (Union of Pure Complex Fuzzy Classes). Let $\Gamma=\left\{V, z, \mu_{\Gamma}(V, z) \mid V \in 2^{U}, z \in U\right\}$ and $\Psi=$ $\left\{T, z, \mu_{\Psi}(T, z) \mid T \in 2^{U}, z \in U\right\}$ be two complex fuzzy classes such that $V$ and $T$ are fuzzy sets. Assume that $\Gamma$ and $\Psi$ are defined over a universe of discourse $U$, and let $2^{U}$ denote the power set of $U$. Further assume that the degree of membership of an object $z \in V$ and an object $y \in T$ is given by $\mu_{\Gamma}(V, z)=\mu_{\Gamma_{r}}(V)+j \mu_{\Gamma_{i}}(z)$ and $\mu_{\Psi}(T, y)=\mu_{\Psi_{r}}(T)+j \mu_{\Psi_{i}}(y)$, respectively, where $\mu_{\Gamma_{r}}(\alpha)$, $\mu_{\Psi_{r}}(\alpha), \mu_{\Gamma_{i}}(\alpha)$, and $\mu_{\Psi_{i}}(\alpha)$ stand for the real and imaginary parts of $\mu_{\Gamma}(V, x)$ and $\mu_{\Psi}(T, y)$. Finally, let $W=2^{U}$, and let $\oplus$ denote a t-conorm operation. Then

$$
\mu_{\Gamma \cup \Psi}(W, z)=\left(\mu_{\Gamma_{r}}(V) \oplus \mu_{\Psi_{r}}(T)\right)+j\left(\mu_{\Gamma_{i}}(z) \oplus \mu_{\Psi_{i}}(z)\right) .
$$

2) Equation for Intersection: The position with respect to intersection is analogous to that of union, and again the definition presented here is described by Tamir et al. as “... sound, intuitive, and practical" [3, pages 301-302].

Definition 8 (Intersection of Pure Complex Fuzzy Classes). Let $\Gamma=\left\{V, z, \mu_{\Gamma}(V, z) \mid V \in 2^{U}, z \in U\right\}$ and $\Psi=$ $\left\{T, z, \mu_{\Psi}(T, z) \mid T \in 2^{U}, z \in U\right\}$ be two complex fuzzy classes such that $V$ and $T$ are fuzzy sets. Assume that $\Gamma$ and $\Psi$ are defined over a universe of discourse $U$, and let $2^{U}$ denote the power set of $U$. Further assume that the degree of membership of an object $z \in V$ and an object $y \in T$ is given by $\mu_{\Gamma}(V, z)=\mu_{\Gamma_{r}}(V)+j \mu_{\Gamma_{i}}(z)$ and $\mu_{\Psi}(T, y)=\mu_{\Psi_{r}}(T)+j \mu_{\Psi_{i}}(y)$, respectively, where $\mu_{\Gamma_{r}}(\alpha)$,

\footnotetext{
${ }^{2}$ Tamir et al. present their analysis in terms of classes, but for the purposes of this paper it can be assumed that classes and sets are equivalent.
} 
$\mu_{\Psi_{r}}(\alpha), \mu_{\Gamma_{i}}(\alpha)$, and $\mu_{\Psi_{i}}(\alpha)$ stand for the real and imaginary parts of $\mu_{\Gamma}(V, x)$ and $\mu_{\Psi}(T, y)$. Finally, let $W=2^{U}$, and let $\odot$ denote a t-norm operation. Then

$$
\mu_{\Gamma \cap \Psi}(W, z)=\left(\mu_{\Gamma_{r}}(V) \odot \mu_{\Psi_{r}}(T)\right)+j\left(\mu_{\Gamma_{i}}(z) \odot \mu_{\Psi_{i}}(z)\right) .
$$

3) Union and Intersection Performed Slice by Slice: Union and intersection operations proceed slice by slice, so it is sufficient to specify how these operations may be applied to two slices. For union, Equation 6 requires that the t-conorm operator be applied to both the real and imaginary components of the membership grade i.e the $\max / \max$ combination. For intersection, Equation 7 indicates that the t-norm operator is applicable to both the real and imaginary components of the membership grade i.e the min / min combination.

\section{B. Operations on Type-2 Fuzzy Sets}

1) Equation for Join: The formula for the join operation is:

$$
\mu_{\tilde{A} \cup \tilde{B}}(x)=\int_{u \in J_{x}^{u}} \int_{w \in J_{x}^{w}} f_{x}(u) \star g_{x}(w) /(u \vee w) \quad x \in X,
$$

where $\vee$ is the maximum operator, $\star$ signifies a t-norm, $\iint$ represents union over $J_{x}^{u} \times J_{x}^{w}$, and $f_{x}(u)$ and $g_{x}(w)$ are respectively the corresponding secondary grades of $\mu_{\tilde{A}}(x)$ and $\mu_{\tilde{B}}(x)$ [5, pages 217-18].

2) Equation for Meet: The formula for the meet operation is:

$$
\mu_{\tilde{A} \cap \tilde{B}}(x)=\int_{u \in J_{x}^{u}} \int_{w \in J_{x}^{w}} f_{x}(u) \star g_{x}(w) /(u \wedge w) \quad x \in X,
$$

where $\wedge$ is the minimum operator, $\star$ signifies a t-norm, $\iint$ represents union over $J_{x}^{u} \times J_{x}^{w}$, and $f_{x}(u)$ and $g_{x}(w)$ are respectively the corresponding secondary grades of $\mu_{\tilde{A}}(x)$ and $\mu_{\tilde{B}}(x)$ [5, page 219].

3) Join and Meet Performed Slice by Slice: Join and meet operations proceed slice by slice, so it is sufficient to specify how these operations may be applied to two slices.

Let $\tilde{A}$ and $\tilde{B}$ be two type- 2 fuzzy sets, in which the codomains are discretised into $N$ slices, and the domains sliced at the points $x_{\tilde{A}}$ and $x_{\tilde{B}}$ respectively. Two type-1 fuzzy sets,

$$
S_{\tilde{A}}=\left\{z_{A_{1}} / y_{A_{1}}+z_{A_{2}} / y_{A_{2}}+\cdots+z_{A_{N}} / y_{A_{N}}\right\}
$$

and

$$
S_{\tilde{B}}=\left\{z_{B_{1}} / y_{B_{1}}+z_{B_{2}} / y_{B_{2}}+\cdots+z_{B_{N}} / y_{B_{N}}\right\},
$$

are generated.

Join: The formula for join requires that all $N^{2}$ possible $\min / \max$ pairings of $S_{\tilde{A}}$ and $S_{\tilde{B}} \quad$ be created: $\min \left(z_{A_{1}}, z_{B_{1}}\right) / \max \left(y_{A_{1}}, y_{B_{1}}\right)+$ $\min \left(z_{A_{1}}, z_{B_{2}}\right) / \max \left(y_{A_{1}}, y_{B_{2}}\right)+\cdots+\min \left(z_{A_{N}}, z_{B_{N}}\right) /$ $\max \left(y_{A_{N}}, y_{B_{N}}\right)$.

Meet: Similarly, for meet, pairings are generated as follows: $\min \left(z_{A_{1}}, z_{B_{1}}\right) / \min \left(y_{A_{1}}, y_{B_{1}}\right)+$ $\min \left(z_{A_{1}}, z_{B_{2}}\right) / \min \left(y_{A_{1}}, y_{B_{2}}\right)+\cdots+\min \left(z_{A_{N}}, z_{B_{N}}\right) /$ $\min \left(y_{A_{N}}, y_{B_{N}}\right)$.
Selection of Maximum Membership Grade: The next stage is the same for join and meet. For every resultant domain value ('denominator') generated, the maximum membership grade ('numerator') is selected. The resultant set of pairs is the join or meet of the two slices.

\section{Applying Join and Meet of Slices to Complex Fuzzy Sets}

What happens when the join and meet operations of type2 fuzzy sets are substituted for the union and intersection operations of (pure) complex fuzzy sets? The complex fuzzy set would be regarded as a type-2 fuzzy set whereby for each vertical slice the co-domain is not discretised i.e. $N=1$. It follows that $N^{2}=1$, indicating that there is only $1 \mathrm{~min} / \max$ pairings of $S_{\tilde{A}}$ and $S_{\tilde{B}}$ in the case of join (union) and only $1 \mathrm{~min} /$ min pairings in the case of meet (intersection). There would be no need to select the maximum membership grade as there would only be one pair; this stage is superfluous. The resultant set of one pair is the join or meet of the two slices.

Is pure complex fuzzy inferencing isomorphic to type-2 fuzzy inferencing? The type- 2 meet operation carries over to the pure complex intersection operation as $\mathrm{min} / \mathrm{min}$. However the type-2 join operation is not transferrable to the pure complex union operation; the former is $\min / \max$, whereas the latter is $\max / \max$. So there is no isomorphism between pure complex fuzzy sets and type-2 fuzzy sets.

\section{Conclusions}

This paper has evaluated the similarities and distinctions between complex fuzzy sets and systems and type-2 fuzzy sets and systems in several respects:

a) Rationale: For a complex fuzzy set, the third dimension reflects additional information - that of phase. However, for a type- 2 fuzzy set, the third dimension reflects the uncertainty arising out of a deficit in information.

b) Applications: Complex fuzzy sets are applicable to the analysis of time series, where there is a phased regularity. In contrast, type- 2 fuzzy sets lend themselves to applications in which there is a high degree of uncertainty, specifically situations where there is uncertainty in multiple dimensions [25].

c) Definitions: Complex fuzzy sets in both their forms differ from type- 2 fuzzy sets in terms of definition. However there is equivalence between the definition of a pure complex fuzzy set and that of a type-2 fuzzy set.

d) Structure: Structural similarity is evident between these three-dimensional fuzzy sets. Complex fuzzy sets are represented by a line in 3D, and type- 2 fuzzy sets by a surface in 3D. However a surface is a generalisation of a line. The similarity between pure complex fuzzy sets and type-2 fuzzy sets is striking, as both are mappings from the domain onto the unit square.

e) Operations: Standard complex fuzzy sets were not compared with type-2 fuzzy sets as regards inferencing operations. A comparison was made of type-2 fuzzy inferencing operations with those of pure complex fuzzy sets; an isomorphism was shown not to exist. 
Overall, the differences outweigh the similarities; despite their shared three-dimensional nature, complex fuzzy sets and type-2 fuzzy sets are two distinct entities, constructed for different purposes, and with different behaviour mathematically.

As further work, it might be profitable to investigate combining (standard) complex fuzzy sets with type-2 fuzzy sets.

\section{REFERENCES}

[1] D. Ramot, R. Milo, M. Friedman, and A. Kandel, "Complex Fuzzy Sets," IEEE Transactions on Fuzzy Systems, vol. 10, no. 2, pp. 171 186, 2002.

[2] S. Dick, "Toward Complex Fuzzy Logic," IEEE Transactions on Fuzzy Systems, vol. 13, no. 3, pp. 405-414, 2005.

[3] D. E. Tamir, L. Jin, and A. Kandel, "A New Interpretation of Complex membership Grade," International Journal of Intelligent Systems, vol. 26, pp. 285-312, 2011.

[4] L. A. Zadeh, "The Concept of a Linguistic Variable and its Application to Approximate Reasoning," Information Sciences, vol. 8, pp. 199 - 249, 1975.

[5] J. M. Mendel, Uncertain Rule-Based Fuzzy Logic Systems: Introduction and New Directions. Prentice-Hall PTR, 2001.

[6] L. A. Zadeh, "Keynote Address," in Proceedings of Joint 9th IFSA World Congress and 20th NAFIPS International Conference, Vancouver, Canada, 2001.

[7] D. R. M. Friedman, G. Langholz, and A. Kandel, "Complex Fuzzy Logic," IEEE Transactions on Fuzzy Systems, vol. 11, no. 4, pp. 450 461, 2003

[8] J. M. Mendel and R. I. John, "Type-2 Fuzzy Sets Made Simple," IEEE Transactions on Fuzzy Systems, vol. 10, no. 2, pp. 117 - 127, 2002.

[9] G. J. Klir and T. A. Folger, Fuzzy Sets, Uncertainty, and Information. Prentice-Hall International, 1992.

[10] O. Castillo and P. Melin, "Optimization of Type-2 Fuzzy Systems Based on Bio-Inspired Methods: A Concise Review," Information Sciences, vol. 205 , pp. 1 - 19, 2012.

[11] J. M. Mendel, "On Answering the Question "Where do I start in order to solve a new problem involving interval type-2 fuzzy sets?"," Information Sciences, vol. 179, no. 19, pp. 3418-3431, 2009.

[12] H. Hagras and C. Wagner, "Introduction to Interval Type-2 Fuzzy Logic Controllers - Towards Better Uncertainty Handling in Real World Applications," IEEE Systems, Man and Cybernetics eNewsletter, 27, 2009.

[13] P. A. Birkin and J. M. Garibaldi, "A Novel Dual-Surface Type-2 Controller for Micro Robots," in Proceedings of FUZZ-IEEE 2010, Barcelona, Spain, 2010, pp. 359-366.

[14] M. Zaher, H. Hagras, A. Khairy, and M. Ibrahim, "A Type-2 Fuzzy Logic based Model for Renewable Wind Energy Generation," in Proceedings of FUZZ-IEEE 2010, Barcelona, Spain, 2010, pp. 511-518.

[15] C. Leal-Ramírez, O. Castillo, P. Melin, and A. Rodríguez-Díaz, "Simulation of the Bird Age-Structured Population Growth based on an Interval Type-2 Fuzzy Cellular Structure," Information Sciences, vol. 181, no. 3, pp. 519-535, 2011.

[16] O. Linda and M. Manic, "Interval Type-2 Fuzzy Voter Design for Fault Tolerant Systems," Information Sciences, vol. 181, no. 14, pp. 2933 2950, July 2011.

[17] O. Castillo and P. Melin, "A Review on the Design and Optimization of Interval Type-2 Fuzzy Controllers," Applied Soft Computing, vol. 12, pp. $1267-1278,2012$.

[18] R. I. John and S. Coupland, "Type-2 Fuzzy Logic: A Historical View," IEEE Computational Intelligence Magazine, vol. 2, no. 1, pp. 57 - 62, 2007.

[19] T. Ozen and J. M. Garibaldi, "Investigating Adaptation in Type-2 Fuzzy Logic Systems Applied to Umbilical Acid-Base Assessment," in Proceedings of 2003 European Symposium on Intelligent Technologies (EUNITE 2003), Oulu, Finland, July 2003, pp. 289-294.

[20] O. Linda and M. Manic, "General Type-2 Fuzzy C-Means Algorithm for Uncertain Fuzzy Clustering," IEEE Transactions on Fuzzy Systems, vol. 20, no. 5, pp. 883 - 897, October 2012.

[21] S. Greenfield, F. Chiclana, R. I. John, and S. Coupland, "The Sampling Method of Defuzzification for Type-2 Fuzzy Sets: Experimental Evaluation," Information Sciences, vol. 189, pp. 77 - 92, 2012.
[22] F. Liu, "An Efficient Centroid Type-Reduction Strategy for General Type-2 Fuzzy Logic System," Information Sciences, vol. 178, no. 9, pp. 2224-2236, 2008.

[23] S.-M. Zhou, F. Chiclana, R. I. John, and J. M. Garibaldi, "Type-1 OWA Operators for Aggregating Uncertain Information with Uncertain Weights Induced By Type-2 Linguistic Quantifiers," Fuzzy Sets and Systems (ISSN 0165-0114), vol. 159, no. 24, pp. 3281 - 3296, 2008.

[24] A. G. B. of Meteorology: Radio and S. W. Services, "Monthly Sunspot Numbers," 2013, http://www.ips.gov.au/Solar/1/6 [Online; accessed 21February-2013].

[25] S. Greenfield and R. I. John, "The Uncertainty Associated with a Type2 Fuzzy Set," in Rudolf Seising (editor) Views on Fuzzy Sets and Systems from Different Perspectives, in 'Studies in Fuzziness and Soft Computing', series editor Janusz Kacprzyk, vol. 243. Springer-Verlag, 2009, pp. 471-483. 